\title{
The Inhibitory Thermal Effects of Focused Ultrasound on an Identified, Single Motoneuron
}

\author{
Morgan N. Collins, ${ }^{1}$ Wynn Legon, ${ }^{2}$ and ${ }^{-K a r e n ~ A . ~ M e s c e ~}{ }^{1,3}$
}

\author{
https://doi.org/10.1523/ENEURO.0514-20.2021
}

${ }^{1}$ Graduate Program in Neuroscience, University of Minnesota, St. Paul, MN 55108, ${ }^{2}$ Department of Neurological Surgery, School of Medicine, University of Virginia, Charlottesville, VA 22901, and ${ }^{3}$ Departments of Entomology and Neuroscience, University of Minnesota, St. Paul, MN 55108

\begin{abstract}
Focused ultrasound (US) is an emerging neuromodulation technology that has gained much attention because of its ability to modulate, noninvasively, neuronal activity in a variety of animals, including humans. However, there has been considerable debate about exactly which types of neurons can be influenced and what underlying mechanisms are in play. Are US-evoked motor changes driven indirectly by activated mechanosensory inputs, or more directly via central interneurons or motoneurons? Although it has been shown that US can mechanically depolarize mechanosensory neurons, there are no studies that have yet tested how identified motoneurons respond directly to US and what the underlying mechanism might be. Here, we examined the effects of US on a single, identified motoneuron within a well-studied and tractable invertebrate preparation, the medicinal leech, Hirudo verbana. Our approach aimed to clarify single neuronal responses to US, which may be obscured in other studies whereby US is applied across a diverse population of cells. We found that US has the ability to inhibit tonic spiking activity through a predominately thermal mechanism. US-evoked effects persisted after blocking synaptic inputs, indicating that its actions were direct. Experiments also revealed that US-comparable heating blocked the axonal conduction of spontaneous action potentials. Finally, we found no evidence that US had significant mechanical effects on the neurons tested, a finding counter to prevailing views. We conclude that a non-sensory neuron can be directly inhibited via a thermal mechanism, a finding that holds promise for clinical neuromodulatory applications.
\end{abstract}

Key words: conduction block; electrophysiology; focused ultrasound; invertebrates; motoneurons; thermal inhibition

\section{Significance Statement}

Much of the enthusiasm regarding focused ultrasound (US) neuromodulation stems from human and other mammalian noninvasive transcranial stimulation and its effects on evoked potentials or motor activity. However, there is considerable debate in the field of US neuromodulation about exactly which types of neurons can be influenced, what the direct outcomes are, and what underlying mechanisms are responsible. In our study, conducted in the medicinal leech Hirudo verbana, we examine for the first time whether a motoneuron could respond to US, which was accomplished at the single-cell level. We found that under conditions whereby US generated sufficient heat $\left(2-3^{\circ} \mathrm{C}\right)$, an inhibitory response was generated. These results have important implications for the noninvasive treatment of chronic pain and other neural disorders.

Received November 27, 2020; accepted March 28, 2021; First published April 14, 2021.

The authors declare no competing financial interests.
Author contributions: M.N.C., W.L., and K.A.M. designed research; M.N.C. performed research; M.N.C. analyzed data; M.N.C. and K.A.M. wrote the paper. 


\section{Introduction}

Focused ultrasound (US) is an emerging neuromodulation technology with the potential to modulate neuronal activity noninvasively and with great precision. Although US's effects on neural tissues have been investigated for nearly a century (Harvey, 1929), renewed interest in US has recently emerged because of the recognized therapeutic value of electrical neuromodulation technologies (Miocinovic et al., 2013; Chakravarthy et al., 2016; Grider et al., 2016). Because US can target deep neural structures noninvasively with accuracy on the order of millimeters (Anderson et al., 1951; Hynynen and Clement, 2007; Legon et al., 2018a), it could provide a viable alternative to implantable neuromodulatory devices, sparing patients the risks and financial burdens of surgery.

Despite the advantages of US, its reported effects are variable. These effects in mammalian systems range from neuronal excitation (Tyler et al., 2008; Tufail et al., 2010; Yoo et al., 2011; Kim et al., 2012, 2014, 2015; Downs et al., 2018) to inhibition (Anderson et al., 1951; Fry et al., 1958; Takagi et al., 1960; Shealy and Henneman, 1962; Rinaldi et al., 1991; Min et al., 2011; Legon et al., 2014). Invertebrate preparations including Caenorhabditis elegans, earthworms and crayfish share similar disparities, with reports of both neuronal excitation (Kubanek et al., 2018; Lin et al., 2019) and inhibition (Wright et al., 2017; Yoo et al., 2017; Zhou et al., 2017).

One potential factor contributing to this variability is the use, by most studies, of response measures of multiunit activity, including compound action potentials (Tsui et al., 2005; Wright et al., 2015; Yoo et al., 2017), event-related potentials (Legon et al., 2014, 2018b; Kim et al., 2015), and BOLD signals (Yoo et al., 2011; Ai et al., 2016, 2018). Population-level measurements can be difficult to interpret, as effects that appear to be direct on target tissues may result from mechanical activation of synapticallycoupled sensory neurons. Examples include auditory hair cells, which can produce widespread cortical activation following US brain application (Guo et al., 2018; Sato et al., 2018), and cells expressing ion channels sensitive to US, including members of the Piezo (Prieto et al., 2018), TRP (Yoo et al., 2020), and DEG/ENaC/ASIC (Kubanek et al., 2018) ion channel families, which are most commonly associated with sensory neurons.

In this study, we sought to determine whether single, non-sensory neurons could directly respond to US, and

This work was supported by a University of Minnesota MnDRIVE Neuromodulation Fellowship (M.N.C.) and by a University of Minnesota grant from the Office of the Vice-President for Research and Grant-in-Aid of Research grant (K.A.M.). This work was also partially supported by the National Science Foundation Grant 1451007 (to K.A.M.).

Acknowledgements: We thank Jerel Mueller, John Basile, and Gerardo Rodriguez for their assistance in obtaining hydrophone characterization of the ultrasound output.

Correspondence should be addressed to Karen A. Mesce at mesce001@ umn.edu.

https://doi.org/10.1523/ENEURO.0514-20.2021

Copyright (C) 2021 Collins et al.

This is an open-access article distributed under the terms of the Creative Commons Attribution 4.0 International license, which permits unrestricted use, distribution and reproduction in any medium provided that the original work is properly attributed. what the mode of action might be. To reduce cell-to-cell variability, minimize potential confounding indirect synaptic effects, and focus on a distinct class of non-sensory cells, we studied the actions of US on a single identified motoneuron, the dorsal longitudinal excitor-3 (DE-3). This neuron's morphology and its physiological activity can be uniquely identified across multiple preparations of the medicinal leech, Hirudo verbana, which has an extensively well characterized and tractable central nervous system (Kristan et al., 2005). To our knowledge, ours is the first study to examine how US directly influences a single motoneuron to determine whether cell types not specialized for mechanosensory transduction would respond to US. Furthermore, our paradigm did not require the removal of nervous tissue from the animal, allowing us to examine the effects of US on a single neuron (specifically its axon) within a functional neural network, as well as avoiding alterations to intrinsic neuronal properties that may occur in culture. This single-cell approach not only enabled us to detect, with precision, whether US was an effective actuator of neuronal change, but enabled us to understand the extent to which its mechanical or thermal properties contributed to the cell's alterations.

\section{Materials and Methods}

\section{Animal preparation and recording substrates}

Hermaphroditic adult leeches ( $H$. verbana) were obtained from Niagara Medical Leeches (Niagara) and housed at room temperature $\left(22-24^{\circ} \mathrm{C}\right)$ in a large tank filled with pond water. Leeches acclimated and maintained at room temperature can remain viable for up to a year or longer as described by others (Harley et al., 2015). Leeches were anaesthetized on ice ( $<5 \mathrm{~min}$ ) before dissection. For intact preparations (all US and control trials), leeches were pinned dorsal-side-up on a porous beeswax dish; dissections were minimal and limited to exposing the targeted dorsal posterior (DP) nerve, which contained the axon of the targeted motoneuron, DE-3. An overview of the neuroanatomy of the leech, the DE-3 motoneuron's spike profile, and experimental paradigm are shown in Figure 1.

For isolated preparations (laser, wire, and low-heat US trials), we removed a portion of the dorsal nerve cord containing three segmental ganglia with attached DP nerves. For laser and wire trials, we pinned the nerve cord dorsal-side-up on a silicone polymer surface (Sylgard, Dow Corning). Low-heat US trials were performed using a latex-bottomed dish over a $500 \mathrm{ml}$ bottle filled with a large sponge and deionized, degassed water $($ depth $=15 \mathrm{~cm}$ ). All preparations were bathed in normal saline during dissection, and either normal or calcium-free saline during experimental trials. Normal saline (adapted from Nicholls and Baylor, 1968) was composed of the following: $115 \mathrm{~mm} \mathrm{NaCl}, 4.0 \mathrm{mM} \mathrm{KCl}, 1.8 \mathrm{mM} \mathrm{CaCl}_{2}, 1.5$ $\mathrm{mm} \mathrm{MgCl}_{2}, 10.0 \mathrm{~mm}$ glucose, and $10.0 \mathrm{~mm}$ Trizma preset crystals ( $\mathrm{pH}$ 7.4). Calcium-free saline was prepared by replacing calcium with equimolar manganese as described (recipe from Olsen and Calabrese, 1996).

\section{Electrophysiology}

Extracellular DE-3 activity was recorded using a suction electrode placed on the distal end of the DP nerve; 
A

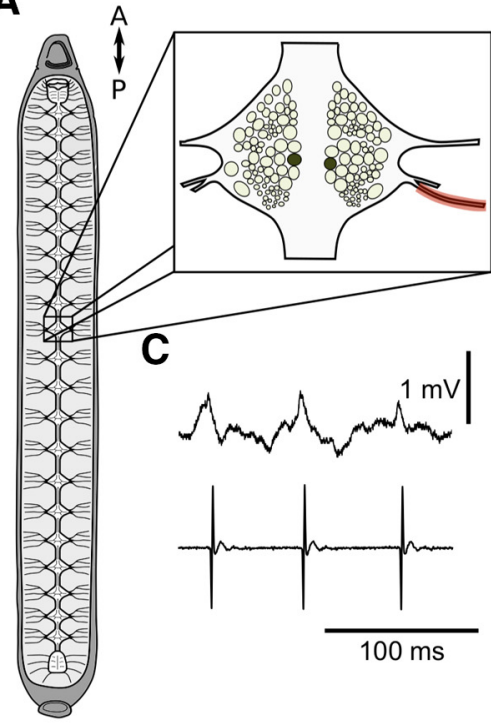

B
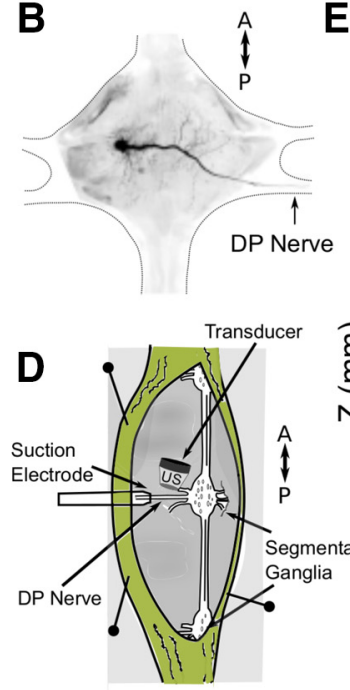

E

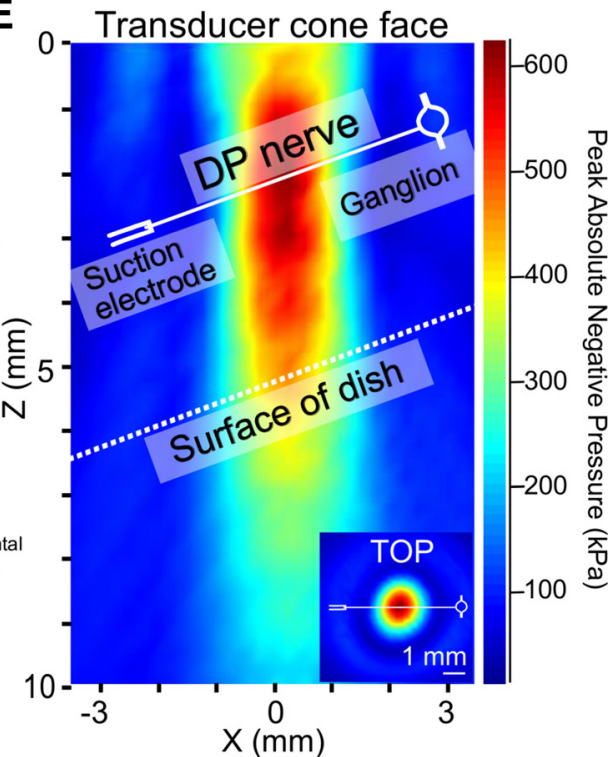

Figure 1. Schematic overview of the experimental preparation (medicinal leech, $H$. verbana), and details of the US transducer and its placement. $\boldsymbol{A}$, Body of the leech dissected open to reveal the CNS, consisting of a cephalic ganglion, 21 individual segmental ganglia and a posterior compound ganglion, all interconnected by longitudinal connectives. Anterior and posterior orientations are indicated by the double-headed arrow (shown throughout). One of the segmental ganglia (ganglion-10) is shown magnified, depicting the bilateral somata located on the dorsal surface of the ganglion. The two filled circles mark locations of the somata of the paired DE-3 motoneurons; one of the two DP nerves (on the right) is tinted red. $\boldsymbol{B}$, A single segmental ganglion (dorsal surface) showing the morphology of the left DE-3 motoneuron obtained by intracellular iontophoretic injection of Neurobiotin. Note: the axon exits the ganglion through the right DP nerve (arrow). A schematized outline of the ganglion and anterior and posterior nerve roots are also shown. $\boldsymbol{C}$, Dual intracellular somatic (top) and extracellular DP nerve (bottom) recordings of spontaneous spiking in the DE3 motoneuron. $\boldsymbol{D}$, Schematic of semi-intact preparation placed in the recording chamber (not to scale) and positioning of the US transducer and suction electrode on the exposed DP nerve. Portrayal of the recording chamber is shown in an artificial upright orientation to better align with the next panel $(\boldsymbol{E})$. $\boldsymbol{E}$, Pressures (in $\mathrm{kPa}$ ) emitted from the face of the US transducer (top of graph). Pressure values (right of graph) are colorized from red (high) to blue (low). Distance from the face of the US transducer is marked in millimeters (left side of graph; 0-mm marks transducer cone face). Hydrophone data (linearly interpolated) are shown at maximum intensity in relationship with the DP nerve and top surface of the recording dish overlaid on scan. US pressures are shown in vertical (i.e., depth) and horizontal (inset) cross-section in relation to the ganglion (white circle, right) and suction electrode (white tubeshape, left). All proportions in $\boldsymbol{E}$ are depicted accurately.

suction electrodes were made in-house, and had a tip diameter of ca. $50 \mu \mathrm{m}$. Signals were amplified by a Model 1700 A-M Systems differential A-C amplifier, and digitized by an Axon CNS Digidata 1440A (Molecular Devices). Intracellular sharp recordings of DE-3 activity were performed using glass electrodes pulled to a resistance of 25-60 M $\Omega$ with a micropipette puller (Sutter Instrument Co, model P-87) and filled with $2 \mathrm{~m}$ potassium acetate. Signals were amplified by an IX2-700 dual intracellular preamp (Dagan Corp.) and digitized as previously described. All signals were recorded with the pClamp software package (Molecular Devices), and imported into MATLAB (MathWorks) for analysis. Extracellular DE-3 activity was identified as the largest spontaneously active unit in the DP recording; somatic intracellular recordings were confirmed to be DE-3 by the cell's size and position, and the correspondence of intracellular and extracellular spikes. The rising phase of the DE-3 extracellularly recorded action potential was typically negative in our recordings; extracellular traces in all figures were inverted for more intuitive viewing. By convention, we have omitted vertical scale bars from extracellular traces because of our use of an AC-coupled amplifier.

\section{Experimental design and statistical analyses}

All trials were performed at room temperature $\left(22-24^{\circ} \mathrm{C}\right)$, the temperature to which the animals had acclimated. Across experiments, we used a total of 106 nerves from 41 hermaphroditic animals (approximately two to three nerves per animal). Sample sizes for heat-only paradigms were $\sim 10$ per condition, consistent with prior leech electrophysiological studies (Puhl and Mesce, 2008; Harley et al., 2015). US and control paradigms had larger sample sizes $(\sim 20)$ to enable subsets of different conditions (e.g., $\mathrm{Ca}^{2+}$ free vs regular salines).

We first tested DP nerves $(N=10)$ with US at five different application durations $(100 \mathrm{~ms}, 316 \mathrm{~ms}, 1.0 \mathrm{~s}, 3.16 \mathrm{~s}$, and $10 \mathrm{~s}$ ). Applications shorter than $10 \mathrm{~s}$ failed to discernibly modulate DE-3 firing rate in any of the nerves tested or evoke firing in other neurons whose axons pass through the DP nerve. To determine whether longer durations would yield more consistent modulation, we extended the application time to $30 \mathrm{~s}$. Our initial attempts suggested that this stimulus duration yielded more reliable and dramatic modulation than shorter durations.

All reported trials were performed on stimulus-naive nerves. Typical trials were $90 \mathrm{~s}$ in duration: $30 \mathrm{~s}$ of 
A
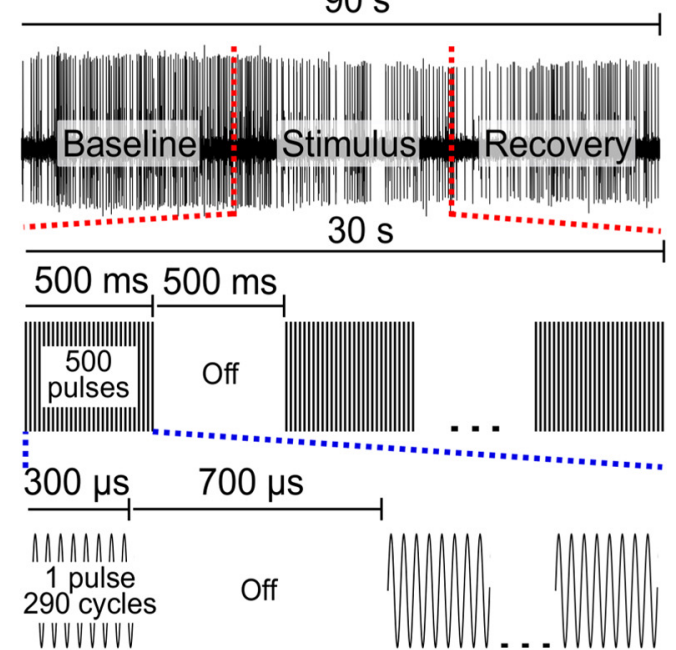

B

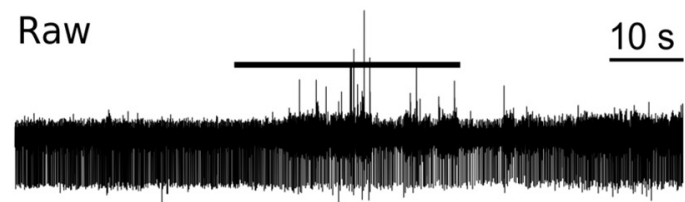

Filtered
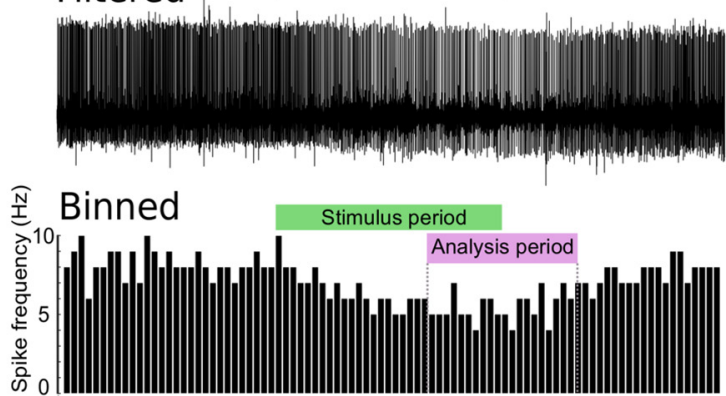

Figure 2. US pulse parameters and trial design with an example response. $\boldsymbol{A}$, Each US application trial lasted $90 \mathrm{~s}$ in duration wherein $960-\mathrm{kHz}$ pulsed US was applied for $30 \mathrm{~s}$, preceded by a baseline period (30 s; example, top trace). Each US pulse was $300 \mu \mathrm{s}$ in duration (bottom trace), and was applied with a 1-kHz pulse repetition frequency with a $50 \%$ duty cycle (middle trace; intrapulse duty cycle of $30 \%$ ). B, Example of extracellular DE-3 recorded spikes with the US-associated artifact (top trace). The same recording after filtering the data with a sixth-order low-pass Butterworth filter (frequency cutoff $=1000 \mathrm{~Hz}$; middle trace). Extracellular spike data from the filtered trace binned in 1-s intervals to yield spike frequency in Hertz (bottom trace). The US application period is denoted by the stimulus period box; the analysis period is denoted by the analysis box.

baseline, $30 \mathrm{~s}$ of stimulus application, and $30 \mathrm{~s}$ of recovery (a sample trial is shown in Fig. 2A). Shorter pulse trials were also $90 \mathrm{~s}$, but with longer recovery periods to compensate for shorter stimulation periods. Recovery periods were extended in trials in which nerves failed to return to within $20 \%$ of baseline firing rate until recovery was achieved, or until sufficient time passed to impair nerve viability $(\sim 1 \mathrm{~h})$. Control trials were equivalent in duration but did not include stimulus application.

Data acquired by pClamp software were imported into MATLAB (R2018b, MathWorks) for all analyses. DE-3 spikes were identified via manually-adjusted thresholding; larger spikes attributed to other cell types (rare) were excluded from analysis via indexing to ensure accurate frequency calculations. DE-3 spikes were binned in 1-s bins for the duration of each trial to yield frequencies in $\mathrm{Hz}$ (Fig. $2 B$ ). Response periods for US were defined as seconds $50-70$ of the trial period (Fig. 2B), as maximal effects were observed starting $20 \mathrm{~s}$ into US application. For the heatonly stimuli, which yielded effects more quickly, the response period was shifted earlier to seconds $40-60$ of the trial period.

Trial data were excluded from analyses if baseline tonic firing was $<1 \mathrm{~Hz}$, or if baseline firing was inconsistent (coefficient of variability $>1$ ); paradigms in which trials were excluded, and final sample sizes, are specified in Results. Mean spike frequencies during the peak response and recovery periods were normalized to $30 \mathrm{~s}$ baseline means for comparison across trials. Averaged responses across trials are reported as percent change from baseline firing rate \pm SE. Nerves were considered responsive if firing rate during the response period differed from baseline by $>20 \%$, the benchmark that encompassed most of the variability in firing in control nerves (Fig. 3B).
All statistical tests were performed in MATLAB with the exception of power analyses, which were performed with G*Power 3.1 (Erdfelder et al., 2009). All tests assumed $\alpha=$ 0.05 . Categorical data were analyzed with Fisher's exact tests. Hypothesis tests were two-tailed Welch's $t$ tests (parametric data) or Kruskal-Wallis tests (non-parametric data). Continuous data were tested for normality with Shapiro-Wilk tests. Correlations are reported as Pearson's $r . R^{2}$ values were obtained with linear regression (least-squares fit). Statistical tests are summarized in Table 1, and are referenced by letters denoting each test described in Results.

\section{US}

We applied 960-kHz US to DP nerves between the ganglion and the suction electrode recording site at the distal end of the DP nerve (Fig. 1D). US was generated with a Sonic Concepts H-102MR transducer coupled with a focusing cone filled with degassed, deionized water. Waveforms were designed by an Agilent 33500B Series function generator and triggered by TTL pulses generated by an Axon CNS Digidata 1440A via pClamp software. Waveforms were amplified by an E\&l 100W RF linear power amplifier (model 2100L), and impedance matched with a Sonic Concepts matching network. US pulses consisted of 290 cycles and were $300 \mu$ s in duration. We applied 500 pulses/s at a $1-\mathrm{kHz}$ pulse repetition frequency; pulse parameters are diagrammed in Figure $2 A$.

Transducer output was characterized by hydrophone (ONDA HNR-0500), as described previously (Collins and Mesce, 2020). Vertical and horizontal cross-sections of linearly interpolated hydrophone measurements at peak amplitudes overlaid with scaled preparation dimensions are shown in Figure $1 E$. 
A

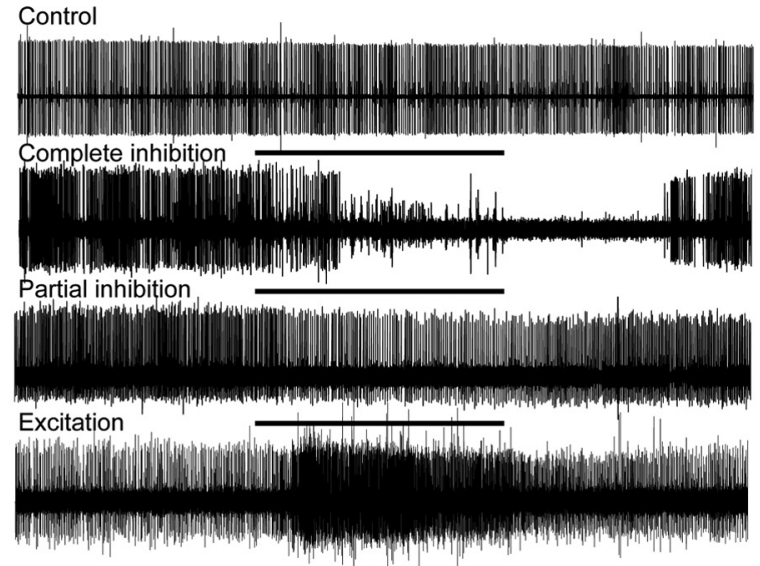

B

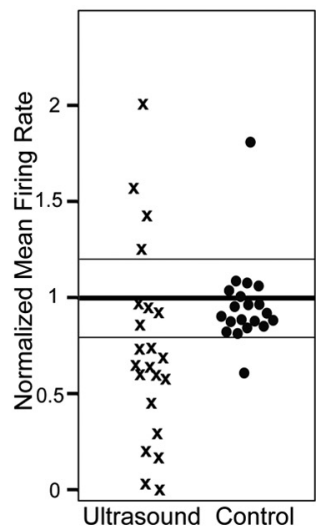

Figure 3. US modulates the activity of motoneuron DE-3. A, Representative extracellular traces of DE-3 spiking activity in the absence of US (top trace) and in response to US applied for $30 \mathrm{~s}$ (horizontal lines denote US application). The predominant response was a reduction in spike activity (two middle traces), and less frequently an increase in spiking activity, sometimes with the recruitment of additional smaller and larger units (bottom trace). US-excited units larger than DE-3 can obscure the DE-3 spike (which is the largest spontaneous unit in the DP nerve), but DE-3's unique amplitude and shape are discernable in expanded traces (data not shown) and quantified in Results. $\boldsymbol{B}$, Scatter plot of normalized mean firing rates during the analysis period of US application and in control trials. The mean of each trial in the study is represented as a single point. Thresholds for "excitatory" and "inhibitory" traces are $20 \%$ above and below baseline mean, as denoted by the thinner horizontal lines. Mean baseline firing rates ranged from 1.0 to $12.1 \mathrm{~Hz}$ (regular US condition) and 1.6 to $10.6 \mathrm{~Hz}$ (control condition).

Absolute peak negative pressure was $\sim 660 \mathrm{kPa}$, yielding a spatial peak pulse average intensity (ISPPA) of $14.52 \mathrm{~W} / \mathrm{cm}^{2}$. Our $30 \%$ duty cycle yielded a spatial peak temporal average intensity (ISPTA) of $4.84 \mathrm{~W} / \mathrm{cm}^{2}$. The transducer was attached to a micromanipulator and positioned such that its peak output was aligned with the center of the $\sim 5$ - $\mathrm{mm}$-long DP nerve. The transducer was tilted at a $20^{\circ}$ angle from vertical to reduce the potential for generation of standing waves.

\section{Heat measurement and apparatuses}

For heat-only experiments, we used two methods of heat application, a $50-\mathrm{mW}$ laser $(650 \mathrm{~nm}$, Visual Fault Locator, J-Deal TL532) with a fiber optic cable attachment (SIMPLEX OS1-9, $125 \mu \mathrm{m}$ in diameter), and a coiled nickel-chromium wire device made in-house and powered by an adjustable direct current source.

In all experiments, the DP nerve was surrounded on all sides by saline. Because of the thinness of the nerve ( $~ 50 \mu \mathrm{m}$ in diameter) and the close similarity of the specific heats and thermal conductivities of water and nervous tissue (Elwassif et al., 2006), we approximated nerve heating by measuring local saline temperature increases with a thermocouple (National Instruments model NIUSBTC01) positioned underneath (in contact with) the DP nerve. Stimuli were applied as described for US and heatonly experiments. Thermocouple measurements were taken at a $1-\mathrm{Hz}$ sampling rate and logged with NCBI thermologger software; data were imported into MATLAB (MathWorks) for plotting and analysis.

\section{Filtering}

US application sometimes caused high-frequency artifact in DP recordings. The amplitude of the artifact was highly variable and was not always resolved or ameliorated with the addition of a bath ground. A digital lowpass Butterworth filter (sampling frequency $=10 \mathrm{kHz}$; cutoff frequency $=1000 \mathrm{~Hz}$; sixth order) was effective in reducing high frequency artifact (Fig. 2B); for consistency, this filter was applied to all traces regardless of stimulus. Beyond high-frequency noise, US application onset and offset were sometimes associated with large-amplitude low-frequency baseline distortions. Affected traces were high pass filtered with a digital Butterworth filter (sampling rate $=10 \mathrm{kHz}$; cutoff frequency $=200 \mathrm{kHz}$ ) to smooth the affected baseline. Residual high-amplitude artifacts were digitally flattened before spike detection to avoid interference; this resulted in a small loss of information ( $0.5 \%$ in noisiest trace). All digital filtering was performed in MATLAB using "butter" and "filter" functions. In addition to filtering, we inverted extracellular traces for more intuitive viewing (the initial vertical deflection from baseline, corresponding to the rising phase of the action potential was made positive).

\section{Results}

\section{The single-cell approach}

Each of the 21 segmental ganglia of the medicinal leech, $H$. verbana, contains a pair of DE-3 motoneurons; each soma is positioned laterally on the dorsal surface of its home ganglion. The DE-3 axon exits each ganglion via the contralateral DP nerve, and its spike is the largest spontaneously active unit in the extracellular DP recording (Ort et al., 1974; Puhl and Mesce, 2008). Importantly, its spontaneous firing property allowed us to examine US's effects on spontaneous versus evoked activity. A diagram of the leech nervous system and an individual ganglion are shown in Figure $1 A$ alongside a Neurobiotin fill of DE3 (Fig. 1B), and representative intracellular and extracellular traces (Fig. 1C). The amplitude of the intracellular 
Table 1: Summary of statistical tests

\begin{tabular}{|c|c|c|c|c|c|}
\hline & Data structure & Type of test & Result & Effect size & Power \\
\hline$a$ & Categorical (binomial) & Fisher's exact test & $p=2.8518 \mathrm{e}-06$ & $\begin{array}{l}\text { Odds } \\
\text { ratio }=40.5\end{array}$ & $95 \% \mathrm{Cl}[6.57,249.65]$ \\
\hline b & $\begin{array}{l}\text { Leech ID: } \\
\text { categorical (nominal) } \\
\text { Mean normalized firing rate during } \\
\quad \text { response period: } \\
\text { normally distributed; } \\
W_{(21)}=0.9469 ; p=0.2353\end{array}$ & One-way ANOVA & $\begin{array}{c}F_{(9,12)}=0.7406 \\
p=0.6686\end{array}$ & $\eta^{2}=0.3571$ & 0.1091 \\
\hline d & $\begin{array}{l}\text { Mean baseline firing rate: } \\
\text { non-normal; } W_{(21)}=0.8446 ; \\
\quad p=0.0040 \\
\text { Mean normalized firing rate during } \\
\text { response period: } \\
\text { normally distributed; } W_{(21)}=0.9469 ; \\
\quad p=0.2353\end{array}$ & $\begin{array}{l}\text { Pearson's } \\
\text { correlation }\end{array}$ & $p=0.2067$ & $r=0.2801$ & $95 \% \mathrm{Cl}[-0.1604,0.6276]$ \\
\hline e & $\begin{array}{l}\text { Mean baseline firing rate: } \\
\text { non-normal; } W_{(21)}=0.8446 ; \\
\quad p=0.0040 \\
\text { Normalized mean absolute } \\
\text { difference from baseline firing rate: } \\
\text { normally distributed; } \\
W_{(21)}=0.9279 ; p=0.1109\end{array}$ & $\begin{array}{l}\text { Pearson's } \\
\text { correlation }\end{array}$ & $p=0.0408$ & $r=-0.4261$ & $\begin{array}{l}95 \% \mathrm{Cl} \\
\quad[-0.7186,-0.0055]\end{array}$ \\
\hline f & $\begin{array}{l}\text { Mean baseline firing rate } \\
\text { in normal saline: } \\
\text { normally distributed; } \\
W_{(12)}=0.9104 ; p=0.1856 \\
\text { Mean baseline firing rate in } \\
\quad \mathrm{Ca}^{2+} \text {-free saline: } \\
\text { non-normal; } W_{(8)}=0.6781 ; p=0.0024\end{array}$ & $\begin{array}{l}\text { Wilcoxon rank-sum } \\
\text { test }\end{array}$ & $p=0.2164$ & $r=0.2635$ & 0.2100 \\
\hline
\end{tabular}
normally distributed;

$W_{(21)}=0.9315 ; p=0.1317$

Coefficient of variability of baseline firing rate in low-heat US trials: normally distributed;

$W_{(19)}=0.9456 ; p=0.3046$

Letters (leftmost column) correspond to statistical tests as reported in Results. The data structure, test type, result, effect size, and statistical power of these tests are described. Where applicable, results of Shapiro-Wilk tests for normality of data are reported under data structure. Effect sizes for Fisher tests are reported as odds ratios. One-way ANOVA effect sizes are reported as $\eta^{2}$, calculated as the between-groups sum of squares divided by the total sum of squares. Effect sizes for Pearson's correlation are the correlation coefficients. The effect size for the Wilcoxon rank-sum test is calculated as the $z$ statistic divided by the square root of the population size, and the effect size of the Welch's $t$ test was calculated as Cohen's $d$ with a correction for small sample sizes as described (Durlak, 2009). When applicable, power was reported as the $95 \%$ confidence interval $(\mathrm{Cl})$ or statistical power calculated post hoc with $\mathrm{G}^{\star} \mathrm{Power}$.

somatic spike is smaller than a typical axonal action potential because of attenuation (via electrotonic spread) from the distal spike-initiating zone; the somata of invertebrate neurons typically have a low density of voltage-gated ion channels (Melinek and Muller, 1996; Stuart, 1970).

\section{US modulates the activity of motoneuron DE-3}

We characterized the effects of 30-s US applications after determining that this stimulus duration yielded more reliable outcomes than shorter durations (see Materials and Methods). To determine the quantitative effects of $30 \mathrm{~s}$ of US on DE-3, we measured the activity of $48 \mathrm{DP}$ nerves $(N=48)$ from 18 leeches. Twenty-six nerves were exposed to 30 s of $960-\mathrm{kHz}$ US; a schematic of an experimental trial is shown in Figure 2. The remaining nerves $(N=22)$ served as untreated controls. Six nerves (four treated with US, two controls) were excluded from analysis because of low spontaneous firing rates $(<1 \mathrm{~Hz} ; N=3$ from US group, $N=1$ from control group) or high firing 
variability (e.g., bursting activity; $N=1$ each from US and control; for exclusion criteria, see Materials and Methods). Thus, $N=22$ nerves exposed to US were subsequently analyzed. Maximal changes in DE-3 firing occurred during the last $10 \mathrm{~s}$ of application and continued for an additional $10 \mathrm{~s}$ (Fig. 2B, analysis box in third trace). Firing rates during this 20-s peak period were normalized to the mean baseline firing rate. Representative traces of US-induced inhibitory and excitatory effects are shown in Figure $3 \mathrm{~A}$ alongside a representative control trial. Normalized means of US-treated and control nerve firing rates during the analysis period are displayed in Figure 3B. Mean deviation from baseline of firing of all US-treated nerves was $47.3 \pm 7.89 \%$ (statistics refer to mean \pm SEM). Mean deviation from baseline of control nerves was $4.56 \pm 5.10 \%$. In control nerves, firing during the analysis window was largely consistent with baseline, with only $2 / 20(10.0 \%)$ having mean firing rates that differed by $>20 \%$. For the US condition, $18 / 22(81.8 \%)$ of treated nerves showed substantial modulation of activity (Fisher's exact test, $p=2.8518 \mathrm{e}-06)^{\mathrm{a}}$. In the US group, we observed mainly inhibitory responses ( 13 out of 18 ; mean $=43.3 \pm 7.63 \%$ decrease in firing rate from baseline). There were a few excitatory cases (four out of 18; mean $=60.7 \pm 15.1 \%$ increase in firing); $1 / 18$ omitted (see Materials and Methods). Some of these excitatory cases in the treated nerves may have been because of some inherent variability across preparations since a similar extent of excitation was also observed across the control nerves. As will be addressed in the following sections, greater excitatory effects may be elicited through US stimulation of presynaptic or synaptic mechanisms rather than direct activation of the soma or axon of a motoneuron; thus, we cannot rule out the possibility that US stimulation was not completely isolated to the DP nerve for the excitatory cases shown in Figure $3 B$.

Finally, as multiple DP nerves were harvested from the same animal, we performed a one-way ANOVA to determine whether normalized mean firing rate during the analysis period was affected by animal. Animal variability did not significantly affect normalized mean firing rate during the response period $\left(F_{(9,12)}=0.7406, p=0.6686\right)^{\mathrm{b}}$, nor did it affect the mean absolute deviation of $D E-3$ firing from baseline during this period $\left(F_{(9,12)}=2.2830, p=0.0918\right)^{C}$.

We also assessed whether the direction of modulation, or the magnitude of modulation, was affected by baseline firing rate. Normalized mean firing rate during the analysis period did not significantly correlate with baseline firing rate (Pearson's correlation, $r=0.2801, p=0.2067)^{\mathrm{d}}$. However, when we tested for correlation between absolute deviation from baseline during the analysis period and baseline firing rate, we found a marginally significant correlation (Pearson's correlation, $r=-0.4261, p=$ $0.0480)^{\mathrm{e}}$, indicating that cells with lower baseline firing rates tended to have greater deviations (either positive or negative) from baseline as a result of US application.

\section{The effects on motoneuron DE-3 are direct and persist during synaptic isolation}

To determine whether US effects were specific to the targeted nerve, a subset $(N=4)$ of nerves tested were accompanied by simultaneous extracellular recordings of DP nerves from adjacent ganglia. DE-3 neurons in neighboring ganglia receive common synaptic inputs, and frequently have similar firing patterns. Three out of four tested nerves responded to US and no comparable effects were observable in the neighboring nerves (Fig. $4 A$, simultaneously recorded traces), suggesting US effects were limited to targeted tissue.

To determine whether observed US actions on DE-3 were direct, or a consequence of activation of synaptically-coupled neurons that may have mechanosensitive properties, a subset of US-treated nerves $(N=10)$ were bathed in calcium-free saline. Calcium was replaced with equimolar manganese, which has been shown to block synaptic transmission in the leech, and which produces less rhythmic oscillatory activity than other replacement divalent cations (Angstadt and Friesen, 1991). This loss of synaptic activity is evidenced by the loss of postsynaptic potentials in intracellular DE-3 recordings (Fig. 4B). Rhythmic firing was observed in one of the ten nerves before US application, and the trial was aborted (final $N=9$ ). A representative trace of US-induced inhibition in $\mathrm{Ca}^{2+}$ free saline is shown in Figure $4 B$. The mean baseline firing rate of DE-3 did not differ between conditions of normal saline and $\mathrm{Ca}^{2+}$-free saline $(3.42 \pm 1.33 \mathrm{~Hz}$; for normal saline: $4.56 \pm 0.57 \mathrm{~Hz} ; p=0.2164$, Wilcoxon rank-sum test $)^{f}$. We observed both excitatory $(N=1)$ and inhibitory $(N=6)$ responses to US within this subset, and a response rate (7/9 nerves, or $77.8 \%)$ matching our overall US sample shown in Figure 3B, suggesting US's effects on DE-3 persist in the absence of synaptic input. Importantly, we observed relatively more inhibition in this condition in comparison to the paradigm using normal saline (summary, Fig. 9), suggesting that some of the excitation we observed in the normal saline condition may have been because of the activation of other neurons, perhaps afferents, that travel in the same DP nerve as does DE-3. The single excitatory case in the $\mathrm{Ca}^{2+}$-free saline is consistent with the potential outlier cases we observed in control nerve experiments; thus, US applied to DE-3 without synaptic input achieves inhibition of firing activity, which is evident beyond spontaneous fluctuations.

\section{Heat mimics US's effects on DE-3}

To determine the magnitude of US-associated tissue heating, we placed a thermocouple directly beneath and in contact with the DP nerve to measure changes in temperature during US application. US induced a temperature increase of $3.42 \pm 0.12^{\circ} \mathrm{C} \quad(n=3$ thermocouple recordings).

Recognizing this increase in nerve temperature could be driving the inhibitory effects, we attempted to minimize the preparation's heating to determine whether effects persisted. We found that our wax substrate contributed to heating by minimizing thermal dissipation. We thus performed US trials on an additional 21 nerves $(N=21)$ on a latex substrate with the recording dish positioned over a large water bath to enable better dissipation of heat (schematic, Fig. 5A). One nerve was excluded from analysis because of high variability in baseline-firing rate (final 

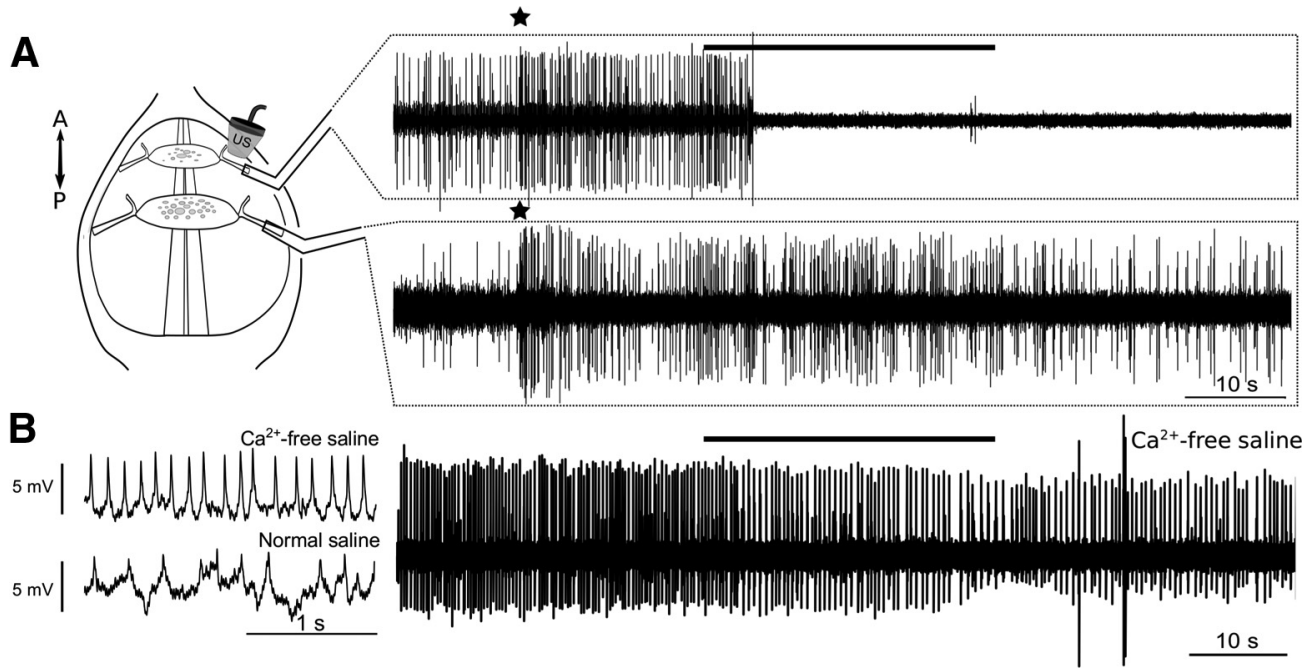

Figure 4. Experiments testing whether US affects the excitability of DE-3 locally and directly. $\boldsymbol{A}$, Schematic of experimental preparation wherein dual extracellular DE-3 recordings were made; dorsal side is up and the anterior-posterior orientation is marked by double-headed arrow. Because the DE-3 motoneurons (and other cells) in adjacent ganglia often receive common synaptic inputs (note: stars indicate an example of shared response), we tested whether US applied to a DE-3 axon in one DP nerve would similarly affect DE-3 and other units in the segmentally adjacent DP nerve (diagram depicting dual DP recordings, left). Dual extracellular recordings from the DP nerves (right) indicate that US inhibition is limited to the DE-3 targeted (upper trace). None of the nerves responding to US (three of four nerves tested) showed a mirrored effect in the adjacent DP nerve. B, Intracellular recordings of spontaneous DE-3 activity in $\mathrm{Ca}^{2+}$-free saline (left, top) and normal saline (bottom, left), showing the reduction of postsynaptic potentials in the absence of $\mathrm{Ca}^{2+}$. Blocking synaptic activity (via bathing in $\mathrm{Ca}^{2+}$ free saline) does not prevent US from inhibiting DE-3 activity (right trace). Of the seven DP nerves responding to US ( $N=10$; one excluded because of bursting), six (85\%) showed an inhibitory response.

$N=20)$. With this paradigm, the temperature increase was limited to $0.3^{\circ} \mathrm{C}$. By greatly reducing heat in this manner, we reduced US modulation (Fig. 5B). Only five of 20 (25\%) DE-3 motoneurons demonstrated more than a $20 \%$ change in firing rate during US application (all inhibited; mean inhibition $=50.9 \pm 5.99 \%$; Fig. $5 \mathrm{C}$ ). Although the number of affected nerves did not differ significantly from control (Fisher's exact test, $p=0.436)^{9}$, a subset of nerves remained susceptible to US modulation despite minimal heating.

We attempted to control further for potential differences associated with our use of different substrates. Standing waves can occur when US reflects off a reflective surface in the direction of the transducer; reflective surfaces are those with a higher acoustic impedance than the surrounding medium, such as our transition from saline to wax. Reflected and emitted waves can summate, causing localized areas of heightened heat and pressure, which have been shown to impact neuronal responsiveness to US by increasing localized radiation force (Menz et al., 2019). Although we had attempted to control for the formation of standing waves by heavily pocking the wax substrate and angling the transducer, as has been shown
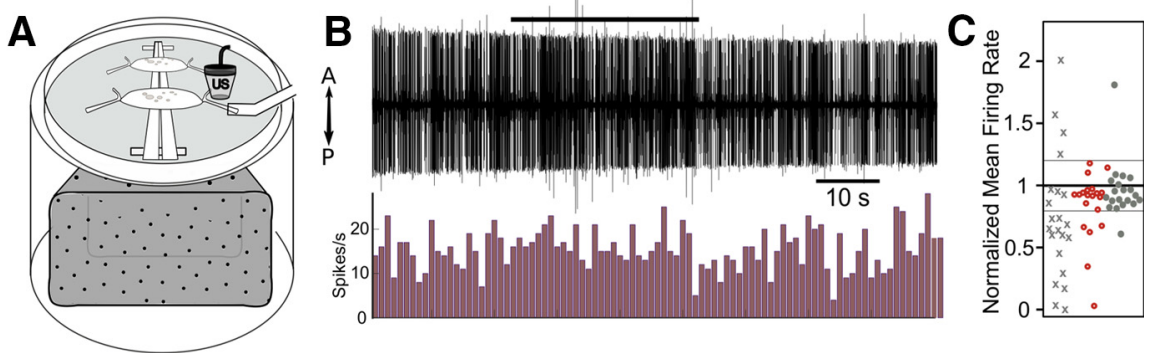

Figure 5. US does not typically modulate neuronal activity in a low-heat paradigm. $\boldsymbol{A}$, Schematic diagram demonstrating the placement of the latex-bottomed dish placed over a water reservoir filled with sponges. Note: ganglia under investigation in this paradigm have been isolated from the body of the leech (see Materials and Methods). Schematic is not shown to scale; reservoir is $\sim 10 \mathrm{~cm}$ in depth. Double-headed arrow indicates anterior-posterior orientation; dorsal side is up. $\boldsymbol{B}$, Representative extracellular trace of DE-3 firing during $30 \mathrm{~s}$ of US (bar) using the latex dish paradigm (upper). Corresponding histogram of spike frequency (lower; bars = 1-s bins). C, Individual mean firing rates of all nerves in low heat US trials during the analysis period (pink circles) shown alongside results from all nerves in regular US trials (gray Xs) and control trials (gray circles). Mean baseline firing rates ranged from 1.0 to $12.1 \mathrm{~Hz}$ (regular US condition), 1.6 to $10.6 \mathrm{~Hz}$ (control condition), and 3.3 to $16.0 \mathrm{~Hz}$ (latex dish condition). 


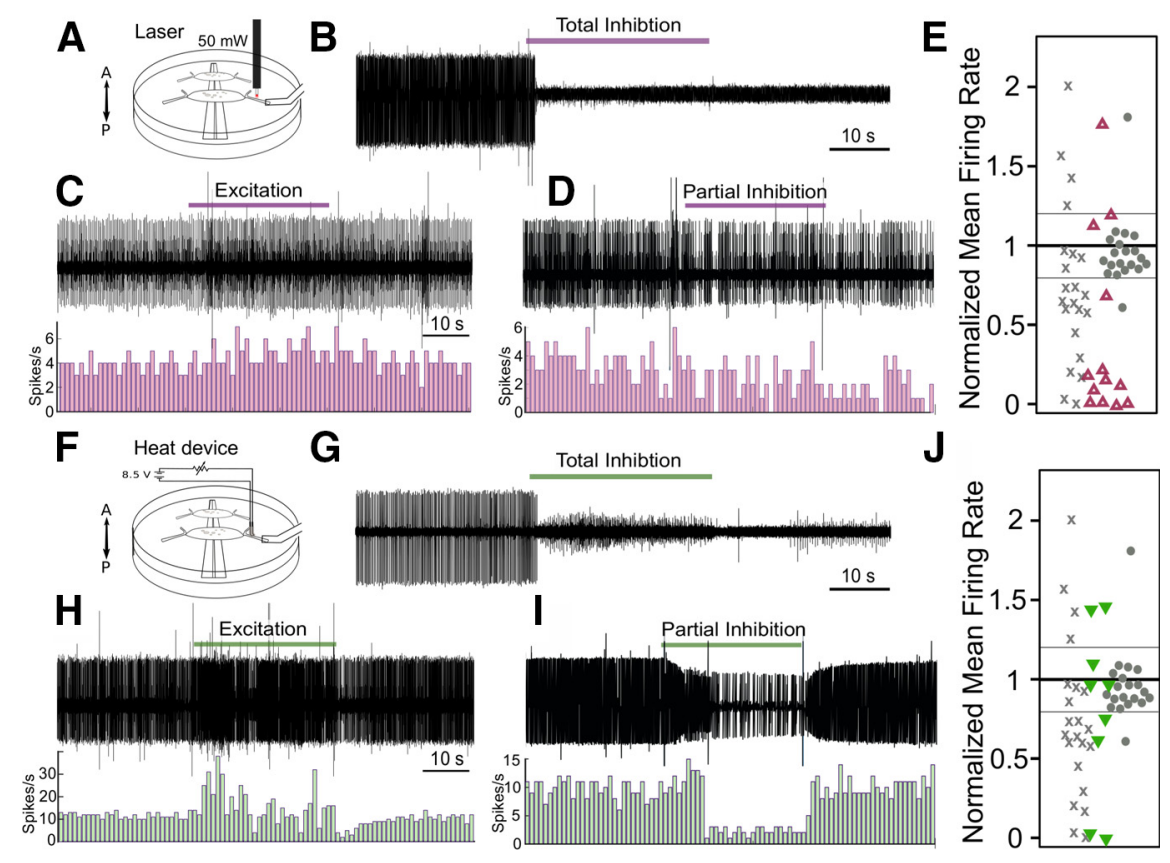

Figure 6. The effects of US can be mimicked by localized application of US-comparable heat. $\boldsymbol{A}$, $\boldsymbol{F}$, Schematics of the laser tool and nichrome wire heating device shown respectively for heat application to the DP nerve. Orientation of ganglia are shown by double-head arrows. B, Representative extracellular trace of DE-3 firing with $30 \mathrm{~s}$ (bar) of thermal stimulation using the laser (50 mW), resulting in total inhibition, the most frequent outcome. As with US, we also observed some excitation $(\boldsymbol{C})$ and partial inhibition $(\boldsymbol{D})$. $\boldsymbol{E}$, Individual mean firing rates of all nerves in laser trials during the analysis period (pink triangles) shown alongside results from all nerves in US trials (gray Xs) and control trials (gray circles). Mean baseline firing rates ranged from 1.0 to $12.1 \mathrm{~Hz}$ (regular US condition), 1.6 to $10.6 \mathrm{~Hz}$ (control condition), and 1.6 to $21.6 \mathrm{~Hz}$ (laser condition). Similar results were obtained using the wire device, with representative traces showing predominantly total inhibition $(\boldsymbol{G})$, excitation $(\boldsymbol{H})$, and partial inhibition (I). $\boldsymbol{J}$, Individual mean firing rates of all nerves in wire device trials during the analysis period (green triangles) shown alongside results from all nerves in US trials (gray Xs) and control trials (gray circles). Mean baseline firing rates ranged from 1.0 to $12.1 \mathrm{~Hz}$ (regular US condition), 1.6 to $10.6 \mathrm{~Hz}$ (control condition), and 6.0 to $15.0 \mathrm{~Hz}$ (wire device condition).

to greatly reduce the neuromodulatory effects of standing waves (Menz et al., 2019), they nevertheless remained a possibility. To ensure our effects with the higher heat paradigm did not stem in part from higher pressures than those used in the lower heat, non-reflective latex dish paradigm, we doubled US absolute peak negative pressure to $1.3 \mathrm{MPa}$ in four nerves in our low-heat latex dish paradigm. None of the four nerves responded to US, suggesting the purely mechanical effects of US at this frequency, if present, were subtle as compared with thermal effects.

We next attempted to replicate the actions of US by inducing comparable US temperature increases in the DP nerve. We found that we could reliably induce a $2.10 \pm 0.017^{\circ} \mathrm{C}(\mathrm{n}=3)$ maximum heat increase in the media surrounding the DP nerve by aiming a $50-\mathrm{mW}$ laser (with a fiber optic attachment) at the nerve for $30 \mathrm{~s}$ at the typical site of US application (schematic, Fig. 6A). We applied the laser to 14 DP nerves from six animals $(N=14)$. One nerve was excluded from analysis because of its high variability in firing rate (final $N=13$ ). Of these nerves, 12 $(92.3 \%)$ had mean firing rates that differed $>20 \%$ from baseline during the 30-s laser application period (Fig. 6E). The laser produced a faster rate of heating than US; peak effects were observed $10 \mathrm{~s}$ into the stimulation and persisted until the end of heat application. Thus, the analysis window was shifted to include data collected during this period (20 s, equivalent to US and control analysis windows). Ten out of 12 responsive DE-3 motoneurons had decreased activity; this inhibition was dramatic (mean = $91.7 \pm 6.48 \%$ ). Two out of 12 were excited (mean = $50.3 \pm 14.21 \%$ increase in firing). Representative traces of neuromodulatory effects are shown in Figure $6 B-D$.

To ensure this laser-induced inhibition stemmed from heating versus a photic mechanism, we performed additional experiments with an alternative heating mechanism: a small insulated nickel-chromium (nichrome) wire coil connected to a direct current source positioned in the typical location of US application (schematic, Fig. 6F). Using the wire heating device, the maximum heat increase of the DP nerve was $4.86 \pm 0.064^{\circ} \mathrm{C}(N=3)$. We tested nine nerves with $30-\mathrm{s}$ applications of heat. As with the laser, the wire heated more quickly than US, and we thus again shifted the analysis window to 40-60 s from trial onset to reflect peak effects. We found that 6/9 (67\%) DE-3 motoneurons had mean firing rates that differed from mean baseline rates by $>20 \%$ (Fig. $6 \mathrm{~J}$ ). Four of six DE-3 motoneurons were inhibited and half of these were completely suppressed (mean inhibition $85.7 \pm 8.0 \%$ ). The remaining two modulated nerves were excited; mean excitation $=29.6 \pm 8.98 \%$. Representative traces of the effects of the wire are shown in Figure 6G-I. 
A

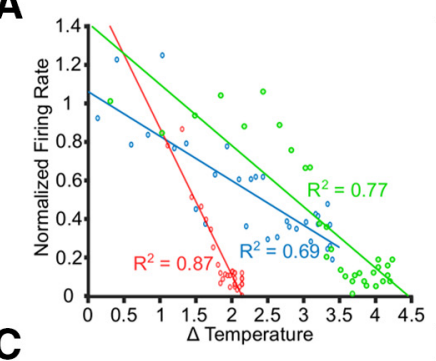

C

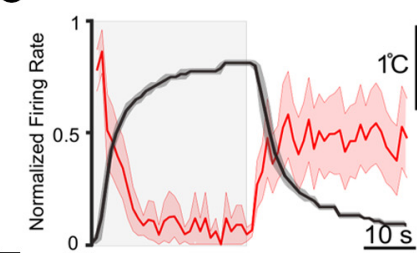

E

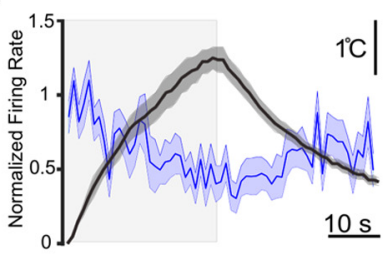

B

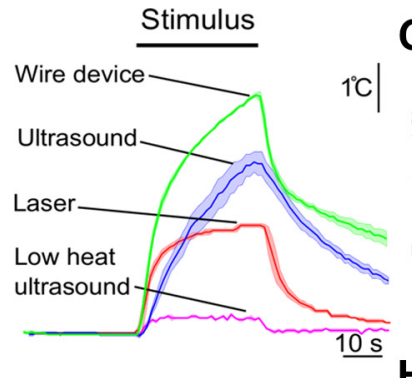

D

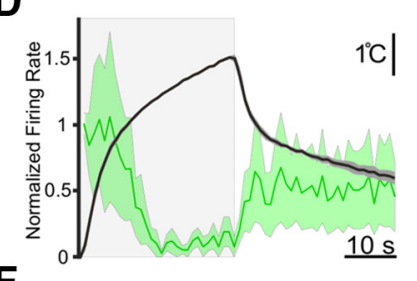

$\mathbf{F}$

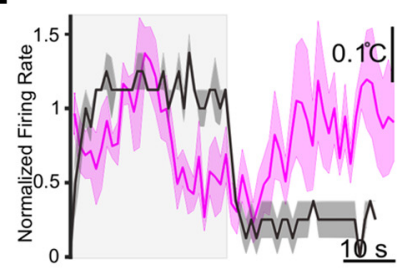
I
G

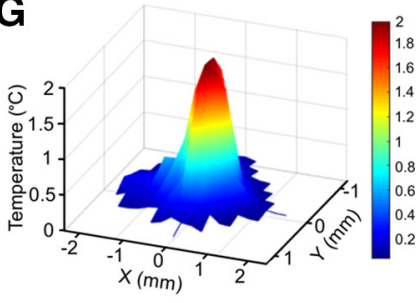

H

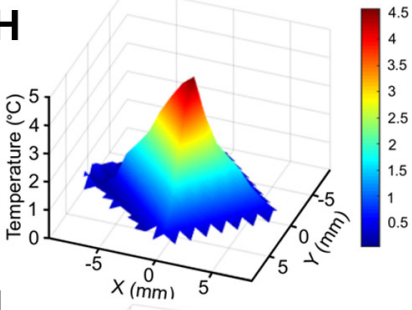

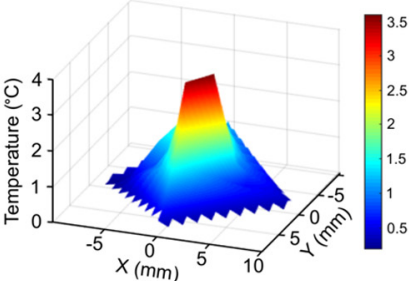

Figure 7. The effects of US can be mimicked by localized application of heat. $\boldsymbol{A}$, Normalized mean firing rates across inhibitory trials (US, laser, wire device) plotted against the corresponding increase in temperature. $\boldsymbol{B}$, Averaged thermocouple recordings $(N=3)$ for each stimulus type; central line $=$ mean, shaded areas $= \pm$ SEM. $\boldsymbol{C}-\boldsymbol{F}$, Averaged normalized firing rates across inhibitory trials. Shaded areas = SEM. Stimulus was applied during gray window. Thermocouple recordings are overlaid (mean $=$ black line, gray shaded area $=$ SEM). G-I, Plots of spatial distribution of heat generated by thermocouple recordings of different stimuli in $x$ and $y$ directions from center (position of nerve). Plots are linearly interpolated from measurements (mean of 2 ) taken at $1 / 4 \mathrm{~mm}$ increments $(\boldsymbol{G})$, or $1 \mathrm{~mm}$ increments $(\boldsymbol{H}, \boldsymbol{I})$; stimuli were attached to a notched micromanipulator to ensure accurate movement, thermocouple remained fixed. $\boldsymbol{G}$, Spatial distribution of heating generated by the laser. $\boldsymbol{H}$, Spatial distribution of heating generated by the wire device. I, Spatial distribution of heating generated by US.

In total, we observed both inhibition and excitation in response to our three stimuli, with a predominance of inhibitory cases. Stimuli ranged in temperature changes from $2.1^{\circ} \mathrm{C}$ to $4.9^{\circ} \mathrm{C}$. In Figure $7 A$, we plotted the firing rates of inhibitory trials for each stimulus that was averaged across trials against increases in temperature, and found a strong correlation for the US, laser, and wire trials (linear regression, least-squares fit, $R^{2}=0.69,0.87$, and 0.77 , respectively). With respect to the low-heat US trials, the correlation between the mean firing rates of inhibitory trials and heating was low $\left(R^{2}=0.11\right)$. The inhibition observed in these trials may have been because of natural variability in firing versus modulation; the baseline mean coefficient of variability in these trials $(0.510 \pm 0.047)$ was slightly higher than in the other US trials $(0.425 \pm 0.049)$, although this difference was not significant $(p=0.222$, Welch's $t$ test) ${ }^{\mathrm{h}}$.

\section{Thermal neuromodulation may be influenced by the spatial spread of heating}

Counter to expectations, the stimulus that generated the smallest temperature increase, the laser, produced the most profound inhibition. While the laser had a sharper rate of heat increase than US, this rate was comparable to that of the wire (Fig. $7 C, F$ ); thus, the magnitude of modulation observed with the laser could not be attributed to the rate of heating alone. We thus investigated whether the area of tissue heated differed between the two types of thermal stimuli. To do so, we measured heat increases from a fixed thermocouple at incremental distances in the $x$ and $y$ directions for all three stimuli. Interpolated plots depicting the spatial spread of heating for each stimulus are shown in Figure 7G-I. While the wire and US had similar heating profiles, with peak heating occurring within a 5-mm radius from the center, the laser produced much more focused heating, with peak heating limited to a $1-\mathrm{mm}$ radius from the center. This restricted heating may have accounted for the relatively greater and less reversible inhibition observed with the laser as compared with the other stimuli. Results by stimulus are summarized in Figure 9.

\section{Poststimulus recovery of US and heat}

Recovery from US and heat application was variable. The firing rates of $14 / 18(77.8 \%)$ US-modulated DE-3 neurons returned to within $20 \%$ of baseline, a value consistent with variations in firing in our control nerves. Recovery typically occurred quickly (mean time to recovery $=21.6 \pm 16.9 \mathrm{~s}$ following the end of stimulation, or $\sim 10 \mathrm{~s}$ after the end of the peak effect period). Excited 


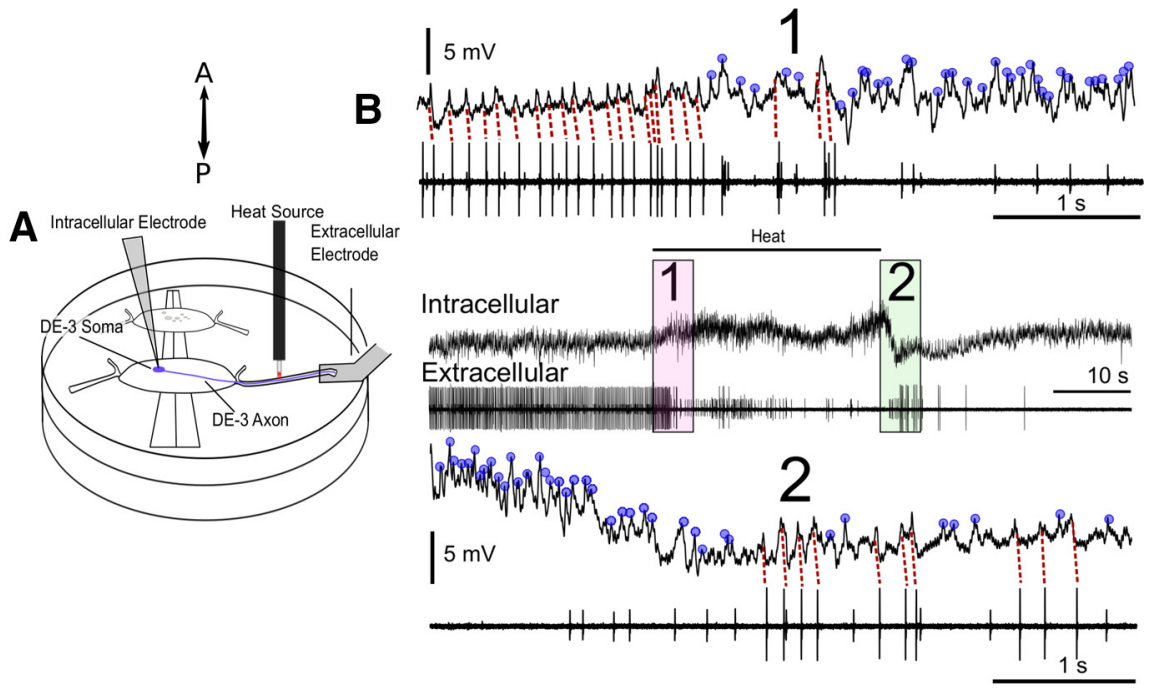

Figure 8. US-comparable heat blocks propagation of the DE-3 spike in the DP nerve. $\boldsymbol{A}$, Schematic showing the placement of heat delivery (laser) and the position of the dual intracellular and extracellular DE-3 recording sites during heat application (red boxes). Double-head arrow indicates orientation of preparation. $\boldsymbol{B}$, At the start of the laser heat application (denoted by horizontal bar), the intracellular spike recorded in the soma of DE-3 (near the spike initiation zone) can be seen to correlate one-for-one with the extracellular DE-3 spike (pink inset 1, expansion of first $5 \mathrm{~s}$ of stimulus). Upon heat delivery, however, the extracellular spike disappears despite the continuation of the intracellular spike (marked with blue dot), indicating a conduction block at the site of heat application between the spike initiation site and the distal nerve (see later portion of pink-inset expansion, top traces). After termination of heat delivery (green inset 2, expansion of $5 \mathrm{~s}$ immediately following the end of the stimulus; bottom traces), partial recovery of the DE-3 axonal spike can be seen. Note (in bottom traces) that waveforms in the intracellular recording are similar whether they are associated with or without their associated axonal spikes (red dotted lines vs blue dots), indicating that we had correctly identified the intracellular activity. In all experiments conducted $(N=3)$, during heat application, the intracellular spike continued in the absence of the mostly silenced axonal spike, which partially recovered after the heat was discontinued.

nerves $(N=4)$ recovered more slowly than inhibited nerves $(N=10 ; 29.3 \pm 13.5$ vs $18.6 \pm 3.70 \mathrm{~s})$. Of the four nerves (all inhibited) that did not return to within $20 \%$ of baseline firing, $2 / 4$ partially recovered $(50.0 \%$ and $74.1 \%$ recovery). The remaining two nerves maintained greatly reduced firing rates for the remainder of the nerve's viability, with one case reaching a maximum of $29.0 \%$ of baseline firing rate $106 \mathrm{~s}$ after the end of the stimulus period, and the other case firing a single time $60 \mathrm{~s}$ after the end of the stimulus. These two minimally recovered nerves were also the most inhibited by US, with $95.1 \%$ inhibition and $100 \%$ inhibition, respectively.

Recovery rates for heat-only stimuli were similar, with $8 / 12(66.7 \%)$ modulated nerves treated with the laser and $4 / 6(66.7 \%)$ nerves treated with the wire returning to within $20 \%$ of baseline firing rate. Mean time to recovery with the laser was $18.8 \pm 8.63 \mathrm{~s}$ $(15.0 \pm 33.5 \mathrm{~s}$ for excited nerves, $22.7 \pm 8.66 \mathrm{~s}$ for inhibited nerves), and $2.75 \pm 1.43 \mathrm{~s}(4.50 \pm 3.50 \mathrm{~s}$ for excited nerves, $1.00 \pm 0.00 \mathrm{~s}$ for inhibited nerves) with the wire. As we observed with US, all nerves that failed to recover fully from heat application had been significantly inhibited (laser: 4/12 nerves, mean inhibition = $99.6 \pm 0.0041 \%$; wire: $2 / 6$ nerves, mean inhibition $=$ $97.2 \pm 0.025 \%)$. Three out of four irreversibly suppressed nerves treated with the laser failed to fire at all poststimulus, as did one of the two nerves irreversibly suppressed with the wire; the other nerves occasionally spiked at rates far below baseline. All nerves that failed to recover were strongly inhibited by stimuli; however, not all strongly inhibited nerves failed to recover. Two nerves whose firing was completely suppressed (100\%) by the laser fully recovered, suggesting total suppression need not be irreversible. Differences in recovery rates may have been because of subtle differences in the placement of the stimulus with respect to the nerve, or other stochastic factors beyond the scope of the present study.

\section{Heat induces conduction block in motoneuron DE-3}

To determine whether the inhibitory effects of US were because of a broad hyperpolarization of DE-3, or from a local conduction block at the site of stimulus application, we performed intracellular somatic recordings of DE-3 in conjunction with application of the laser placed distally on the DP nerve $(N=3)$. The laser was the most compact heat apparatus, and the most compatible with our intracellular electrode placement. Heat was applied between the somatic intracellular electrode and the distal suction electrode (schematic, Fig. 8A). DE-3 activity could thus be measured on either side of the heat stimulus. Figure $8 B$ shows a representative simultaneous intracellular and extracellular recording of the DE-3 motoneuron with an inhibitory response with laser stimulation. Spikes initiated near the soma as measured via our intracellular electrode failed to propagate to the distal electrode because of a presumed conduction block at the site of heat application. 


\begin{tabular}{l} 
Wire device \\
\cline { 2 - 7 }
\end{tabular}

Figure 9. Neuromodulatory actions by stimulus mode. Neuromodulatory effects were found to differ with respect to the spatial properties of stimuli. We observed a trend whereby a broader heat application (e.g., wire device, bath) elicited proportionally more excitation, whereas a narrower heating, including the most focused heat source (i.e., the laser), produced more inhibition. US applied in the $\mathrm{Ca}^{2+}$-free condition (blockage of synaptic communication) elicited relatively more neuronal inhibition compared with applications in regular saline. While the thermal properties in this condition were identical to those in the regular saline one, we observed less excitation, which was likely because of the reduction of network-level synaptic inputs that might increase neuronal activity.

\section{Local versus global heating biases the neuromodulation outcome}

To determine whether a global temperature shift of a comparable magnitude over a similar time course (several seconds) could inhibit firing to the extent of focal heating, we raised the bath temperature by $2^{\circ} \mathrm{C}$ through the rapid addition of heated saline. We found a moderate and short-lived increase in DE-3 firing associated with the addition of heated saline in the four nerves tested $(N=4)$. This effect was comparable to excitatory effects observed in similar bath-heating experiments performed with this preparation (Romanenko et al., 2014). We thus propose that non-noxious thermal inhibitory neuromodulation is only achievable with focused applications of heat, as summarized in Figure 9 based on the combined results presented across our different US and heating experiments.

\section{Discussion}

\section{Brief overview}

In this study, we examined the effects of $30 \mathrm{~s}$ of pulsed 960-kHz US on the axon of motoneuron DE-3, a uniquely identified cell in the medicinal leech. Experiments revealed that the primary effect of US, at these parameters, was suppression of neuronal firing via action potential conduction block. A benefit of our study was that response-type variability (i.e., excitatory vs inhibitory) was confined to the same identified neuron, enabling us to avoid confounding results stemming from any inconsistent access to the same or similar types of neurons across recording sessions, which has been problematic in other invertebrate and mammalian studies. Furthermore, by chemically removing synaptic inputs, we could determine whether stimulus-induced outcomes were a function of direct actions on the targeted motoneuron (Fig. 4).

In contrast to achieving both US-induced and US-comparable heat-induced neuronal inhibition, neuronal excitation was difficult to achieve and deemed more likely dependent on synaptic inputs that were indirectly affected, consistent with previous studies performed in intact brain preparations from mammalian species (Guo et al., 2018; Sato et al., 2018).

\section{Support for a thermal mechanism of US action}

Our data support the idea that US modulates motoneuronal activity via a predominantly, if not entirely, thermal mechanism. We arrived at this conclusion in two ways. First, we were unable to modulate DE-3 neuronal activity reliably in the absence of heat (Fig. 5). Second, we performed additional experiments with a $50-\mathrm{mW}$ laser and a wire device, which mimicked US-associated nerve heating, and found that they could reliably mimic the effects of US (Fig. 6). Results from prior studies examining other types of neurons have come to similar conclusions that the heat component of US drives inhibitory responses (Lele, 1963; Ueda et al., 1977; Darrow et al., 2019).

Short applications of US ( $100 \mathrm{~ms}, 3.16 \mathrm{~s})$, which did not generate significant heating, failed to inhibit or evoke activity. Furthermore, after significantly reducing US-associated heat from our longer applications $(30 \mathrm{~s})$ from $3.5^{\circ} \mathrm{C}$ to $0.3^{\circ} \mathrm{C}$ with a less insulating dish substrate, the rate of neuronal inhibition was reduced substantially from 14/22 nerves to $5 / 20$ nerves. These five remaining recordings may have reflected natural variation in firing, as their mean firing rate in inhibitory trials failed to correlate with changes in temperature $\left(R^{2}=0.11\right)$, as compared with the heat-only and higher heat US paradigms $\left(R^{2}=0.82\right.$, 0.87 , and 0.77 , for higher heat US, laser, and wire, respectively).

It is noteworthy that we observed the least amount of neuronal excitation in trials performed in $\mathrm{Ca}^{2+}$-free saline ( $1 / 9$ nerves) and with the laser (1/13 nerves). $\mathrm{Ca}^{2+}$-free saline prevented sensory cells or other tissues from synaptically exciting DE-3. The laser yielded the most spatially restricted heating, thus limiting the contributions of other neural pathways that may have provided excitatory inputs to the motoneuron as compared with when less focused heat stimuli were used (laser vs wire). These data suggest 
that thermal excitation stems largely or entirely from circuit-level heating, while targeted axonal heating results in inhibition (summarized in Fig. 9). These conclusions are further supported by our inability to generate neuronal inhibition via bath heating of $2^{\circ} \mathrm{C}$, as previously reported in the leech (Romanenko et al., 2014). Finally, the rate and magnitude of inhibition observed, across the different stimuli used, likely stem from differences in the rates of heating (Fig. 7). The rise rate of tissue heating is a salient determinant of neuromodulation outcomes in other forms of thermal neuromodulation, including infrared (Shapiro et al., 2012).

\section{Consideration of non-thermal components of US on DE-3 activity}

Previous studies have proposed that non-thermal mechanisms underlie changes in US-induced neuronal excitation or inhibition, such as intramembrane cavitation (Plaksin et al., 2014) or other mechanical effects, including activation of mechanosensitive ion channels, especially in mechanosensory neurons (Kubanek et al., 2018), and radiation force. Several studies have reported that US at frequencies $<400 \mathrm{kHz}$ more efficiently evoke activity than US at higher frequencies (King et al., 2013; Kim et al., 2014). This discrepancy may be driven by a cavitationbased mechanism, as lower frequencies generate cavitational forces more effectively (Gaertner, 1954). We opted to use higher frequencies $(960 \mathrm{kHz})$ to permit more precise targeting of DE-3 (Carovac et al., 2011). This frequency, however, may be too high to generate cavitational actions. In a comparable preparation (invertebrate nerve), cavitation-evoked potentials could be achieved at $0.67 \mathrm{kHz}$ but not 1.1. $\mathrm{MHz}$ (Wright et al., 2017). However, given its potential to rupture neuronal membranes (Wright et al., 2017), it remains unclear whether this is a desirable application mode to pursue.

Another factor contributing to our inability to evoke activity mechanically may have been the $660-\mathrm{kPa}$ peak pressure we used. This amount of pressure, however, was significantly higher than levels used in studies of cortical brain neurons, which have attributed US effects to mechanical forces (for example, Tyler et al., 2008; Tufail et al., 2010). Furthermore, it is unlikely that we overshot an effective range of pressures because studies reporting mechanically-attributed transcranial effects of US have covered a range of pressures encompassing ours $(0.03-$ $1.11 \mathrm{MPa}$ ), and although there is a saturation point with increasing amplitude, there is no associated decline in US responses (King et al., 2013).

Transcranial US stimulation typically involves the modulation of neuronal somata, whereas, in our study, we targeted a neuron's axon within a peripheral nerve. Thus, one must still consider whether we failed to use a sufficiently high pressure in the context of peripheral nerve activation. This distinction is an important one to consider because peripheral nerves are believed to have higher US activation thresholds than central neural tissues (Wright et al., 2017). Some recent studies of peripheral nerves have revealed that high pressures, far in excess of ours, are needed to evoke motor-related responses; for example,
$2 \mathrm{MPa}$ in invertebrates (Wright et al., 2017), and up to 5.4 MPa (Downs et al., 2018), 11.8 MPa (Kim et al., 2020), and $30 \mathrm{MPa}$ (Lee et al., 2020) in mammals. Thus, it may be possible to evoke mechanically-induced effects with higher US pressures. However, pressures in the 10 s of $\mathrm{MPa}$ range generate intensities that far exceed current safety thresholds for diagnostic use (FDA, 2019), and are likely too destructive for use in reversible neuromodulatory therapies. For reference, the temperature increases in our study $\left(<5^{\circ} \mathrm{C}\right)$ have been shown to be safe in mammalian systems for brain-exposure durations $\leq 60 \mathrm{~min}$ (Haveman et al., 2005). In addition, it is encouraging that $100 \%$ of the nerves we treated with US remained capable of transmitting DE-3 action potentials, with $78 \%$ returning to baseline firing rates within $20 \mathrm{~s}$ of stimulus cessation.

The mechanisms underlying thermal inhibition below the range of temperatures known to cause protein degeneration or necrosis $\left(\sim 45^{\circ} \mathrm{C}\right.$ in humans, or $\sim 8^{\circ} \mathrm{C}$ above normal; Wang et al., 2014) are not completely understood, but may include changes in ion-channel-gating kinetics and conductances. We investigated how US inhibited neural activity thermally at relatively low temperatures $\left(<5^{\circ} \mathrm{C}\right)$. As a proxy for US-associated heat, we used the laser, as it was the most compatible with our intracellular recording electrode, and did not generate electrode resonance as does US, which can obscure the fidelity of intracellular recording data (Collins and Mesce, 2020). During heat application, we observed a continuation of spikes recorded in the soma with a loss of spikes distal to the stimulus (Fig. 8), indicating that the inhibition was because of a failure of spike conduction.

\section{Potential thermal-mediated mechanisms underlying DE-3 inhibition}

One promising mechanism to explain the heat-mediated conduction block we observed is a loss of ion homeostasis. It has been shown that thermal suppression of neural activity is accompanied by a spike in extracellular potassium in invertebrate (Money et al., 2009) and mammalian (Wu and Fisher, 2000) systems. At the circuit level, increased $\left[\mathrm{K}^{+}\right]_{0}$ is believed to underlie spreading depression (Kraio and Nicholson, 1978; Somjen, 2001; Ayata and Lauritzen, 2015), a conserved phenomenon in which neural activity is disrupted until concentration gradients are restored (Spong et al., 2016). Importantly, two earlier studies in rat brain found that US can induce spreading depression, resulting in effects reminiscent of pharmacologically raising extracellular potassium (Koroleva et al., 1986) and increasing temperature (Ueda et al., 1977). Spreading depression-associated inhibition can also be preceded by depolarization of the resting membrane potential (Pietrobon and Moskowitz, 2014), and by hyperexcitation (Rodgers et al., 2007). This mechanism thus might explain the brief uptick in firing rate that preceded some of our inhibitory trials, particularly those using the wire (the "hottest" stimulus in the present study), as evidenced by an initial increase in mean firing rate (Fig. 6).

One source of increased $\left[\mathrm{K}^{+}\right]_{0}$ may be an increased conductance through voltage-gated potassium channels $\left(\mathrm{K}_{\mathrm{V}}\right)$. In Aplysia, heat-mediated (infrared) conduction block 
is greatly reduced by tetraethylammonium (TEA), a $\mathrm{K}_{v}$ antagonist (Ganguly et al., 2019a,b). An additional primary or complementary source of $\left[\mathrm{K}^{+}\right]_{0}$ may be via two-pore potassium channels, which are thermosensitive (Schneider et al., 2014), and whose conductance increases on exposure to US (Kubanek et al., 2016) by a reportedly thermal mechanism (Prieto et al., 2020). Importantly, both classes of potassium channels are expressed ubiquitously by neurons, and a mechanism targeting these channels would circumvent the need to limit US-based neuromodulation therapies to classes of cells that express ion channels susceptible to US mechanical activation, including Piezo (Prieto et al., 2018), TRP (Yoo et al., 2020), and DEG/ENaC/ASIC (Kubanek et al., 2018), or to introduce non-endogenous mechanosensitive ion channels in desired target tissue a la sonogenetics (Ibsen et al., 2015). Furthermore, given safety constraints associated with high pressures that may otherwise be required to modulate non-sensory neurons mechanically, thermal applications may be more practical and versatile than mechanical ones.

\section{Clinical applications}

The ability to suppress neuronal activity safely and reversibly could have a significant clinical impact on a wide range of neurologic disorders. The relevance of the results of this study to human health applications is somewhat tempered by inherent differences between mammalian and invertebrate nervous systems, perhaps the most significant of which is the lack of myelination of invertebrate axons. Despite this key difference, action potential conduction in the leech, which was proposed to be thermally inhibited in this study, is governed by the same classes of ion channels that conduct action potentials in mammals, including a rising phase mediated by voltage-gated sodium channels, and a falling phase mediated by $\mathrm{K}_{\mathrm{V}} \mathrm{s}$ (Kleinhaus, 1976; Kleinhaus and Prichard, 1976). The distribution pattens of these ion channels, across myelinated mammalian nerves and invertebrate fibers, could result in varying outcomes. However, our results are clearly relevant to the modulation of $\mathrm{C}$-fibers involved in the transmission of pain (Costigan and Woolf, 2000), which like invertebrate nerves are unmyelinated. Based on the results of our study, thermal US may be an effective treatment, not only for pain, but for managing excessive peripheral nerve activity, including peripheral neuropathies (St. John Smith, 2018) and spasticity (Raghavan, 2018).

\section{References}

Ai L, Mueller JK, Grant A, Eryaman Y, Legon W (2016) Transcranial focused ultrasound for BOLD fMRI signal modulation in humans. Conf Proc IEEE Eng Med Biol Soc 2016:1758-1761.

Ai L, Bansal P, Mueller JK, Legon W (2018) Effects of transcranial focused ultrasound on human primary motor cortex using 7T fMRI: a pilot study. BMC Neurosci 19:56.

Anderson TP, Wakim KG, Herrick JF, Bennett WA, Krusen FH (1951) An experimental study of the effects of ultrasonic energy on the lower part of the spinal cord and peripheral nerves. Arch Phys Med Rehabil 32:71-83.
Angstadt JD, Friesen WO (1991) Synchronized oscillatory activity in leech neurons induced by calcium channel blockers. J Neurophysiol 66:1858-1873.

Ayata C, Lauritzen M (2015) Spreading depression, spreading depolarizations, and the cerebral vasculature. Physiol Rev 95:953-993.

Carovac A, Smajlovic F, Junuzovic D (2011) Application of ultrasound in medicine. Acta Inform Med 19:168.

Chakravarthy K, Nava A, Christo PJ, Williams K (2016) Review of recent advances in peripheral nerve stimulation (PNS). Curr Pain Headache Rep 20:60.

Collins MN, Mesce KA (2020) Focused ultrasound neuromodulation and the confounds of intracellular electrophysiological investigation. eNeuro 7:ENEURO.0213-20.2020.

Costigan M, Woolf CJ (2000) Pain: molecular mechanisms. J Pain 1:35-44.

Darrow DP, O’Brien P, Richner TJ, Netoff TI, Ebbini ES (2019) Reversible neuroinhibition by focused ultrasound is mediated by a thermal mechanism. Brain Stimul 12:1439-1447.

Downs ME, Lee SA, Yang G, Kim S, Wang Q, Konofagou EE (2018) Non-invasive peripheral nerve stimulation via focused ultrasound in vivo. Phys Med Biol 63:e035011.

Durlak J (2009) How to select, calculate, and interpret effect sizes. J Pediatr Psychol 34:917-928.

Elwassif MM, Kong Q, Vazquez M, Bikson M (2006) Bio-heat transfer model of deep brain stimulation-induced temperature changes. $J$ Neural Eng 3:306-315.

Erdfelder E, Faul F, Buchner A, Lang AG (2009) Statistical power analyses using $G^{*} P o w e r$ 3.1: tests for correlation and regression analyses. Behav Res Methods 41:1149-1160.

FDA (2019) Marketing clearance of diagnostic ultrasound systems and transducers-guidance for industry and food and drug administration staff. US Dep Heal Hum Serv Food Drug Adm Cent Devices Radiol Heal 18-34.

Fry FJ, Ades HW, Fry WJ (1958) Production of reversible changes in the central nervous system by ultrasound. Science 127:83-84.

Gaertner W (1954) Frequency dependence of ultrasonic cavitation. J Acoust Soc Am 26:977-980.

Ganguly M, Ford JB, Zhuo J, McPheeters MT, Jenkins MW, Chiel HJ, Jansen ED (2019a) Voltage-gated potassium channels are critical for infrared inhibition of action potentials: an experimental study. Neurophotonics 6:1.

Ganguly M, Jenkins MW, Jansen ED, Chiel HJ (2019b) Thermal block of action potentials is primarily due to volage-dependent potassium currents: a modeling study. J Neural Eng 16:036020.

Grider JS, Manchikanti L, Carayannopoulos A, Sharma ML, Balog CC, Harned ME, Grami V, Justiz R, Nouri K, Hayek SM, Vallejo R, Christo PJ (2016) Effectiveness of spinal cord stimulation in chronic spinal pain: a systematic review. Pain Phys 19:E33-E54.

Guo H, Hamilton M, Offutt SJ, Gloeckner CD, Li T, Kim Y, Legon W, Alford JK, Lim HH (2018) Ultrasound produces extensive brain activation via a cochlear pathway. Neuron 98:1020-1030.

Harley CM, Reilly MG, Stewart C, Schlegel C, Morley E, Puhl JG, Nagel C, Crisp KM, Mesce KA (2015) Compensatory plasticity restores locomotion after chronic removal of descending projections. J Neurophysiol 113:3610-3622.

Harvey EN (1929) The effect of high frequency sound waves on heart muscle and other irritable tissues. Am J Physiol Content 91:284290.

Haveman J, Sminia P, Wondergem J, van der Zee J, Hulshof MCCM (2005) Effects of hyperthermia on the central nervous system: what was learnt from animal studies. Int J Hyperther 21:473-487.

Hynynen K, Clement G (2007) Clinical applications of focused ultrasound - the brain. Int J Hyperth 23:193-202.

Ibsen S, Tong A, Schutt C, Esener S, Chalasani SH (2015) Sonogenetics is a non-invasive approach to activating neurons in Caenorhabditis elegans. Nat Comm 6:1-12.

Kim H, Taghados SJ, Fischer K, Maeng LS, Park S, Yoo SS (2012) Noninvasive transcranial stimulation of rat abducens nerve by focused ultrasound. Ultrasound Med Biol 38:1568-1575. 
Kim H, Chiu A, Lee SD, Fischer K, Yoo SS (2014) Focused ultrasound-mediated non-invasive brain stimulation: examination of sonication parameters. Brain Stimul 7:748-756.

Kim H, Park MY, Lee SD, Lee W, Chiu A, Yoo SS (2015) Suppression of EEG visual-evoked potentials in rats through neuromodulatory focused ultrasound. Neuroreport 26:211-215.

Kim MG, Kamimura HAS, Lee SA, Aurup C, Kwon N, Konofagou EE (2020) Image-guided focused ultrasound modulates electrically evoked motor neuronal activity in the mouse peripheral nervous system in vivo. J Neural Eng 17:e026026.

King RL, Brown JR, Newsome WT, Pauly KB (2013) Effective parameters for ultrasound-induced in vivo neurostimulation. Ultrasound Med Biol 39:312-331.

Kleinhaus A (1976) Divalent cations and the action potential of leech Retzius cells. Pflugers Arch 363:97-104.

Kleinhaus A, Prichard J (1976) Sodium dependent tetrodotoxin-resistant action potentials in a leech neuron. Brain Res 102:368-373.

Koroleva VI, Vykhodtseva NI, Elagin VA (1986) Cortical and subcortical spreading depression in rats produced by focused ultrasound. Neurophysiology 18:43-48.

Kraio RP, Nicholson C (1978) Extracellular ionic variations during spreading depression. Neuroscience 3:1045-1059.

Kristan WB, Calabrese RL, Friesen WO (2005) Neuronal control of leech behavior. Prog Neurobiol 76:279-327.

Kubanek J, Shi J, Marsh J, Chen D, Deng C, Cui J (2016) Ultrasound modulates ion channel currents. Sci Rep 6:24170.

Kubanek J, Shukla P, Das A, Baccus SA, Goodman MB (2018) Ultrasound elicits behavioral responses through mechanical effects on neurons and ion channels in a simple nervous system. J Neurosci 38:3081-3091.

Lee SA, Kamimura HAS, Burgess MT, Konofagou EE (2020) Displacement imaging for focused ultrasound peripheral nerve neuromodulation. IEEE Trans Med Imaging 39:3391-3402.

Legon W, Sato TF, Opitz A, Mueller J, Barbour A, Williams A, Tyler WJ (2014) Transcranial focused ultrasound modulates the activity of primary somatosensory cortex in humans. Nat Neurosci 17:322-329.

Legon W, Ai L, Bansal P, Mueller JK (2018a) Neuromodulation with single-element transcranial focused ultrasound in human thalamus. Hum Brain Mapp 39:1995-2006.

Legon W, Bansal P, Tyshynsky R, Ai L, Mueller JK (2018b) Transcranial focused ultrasound neuromodulation of the human primary motor cortex. Sci Rep 8:10007.

Lele PP (1963) Effects of focused ultrasonic radiation on peripheral nerve, with observations on local heating. Exp Neurol 8:47-83.

Lin X, Qiu Y, Song L, Chen S, Chen X, Huang G, Song J, Chen X, Yang $\mathrm{H}$ (2019) Ultrasound activation of liposomes for enhanced ultrasound imaging and synergistic gas and sonodynamic cancer therapy. Nanoscale Horiz 4:747-756.

Melinek R, Muller KJ (1996) Action potential initiation site depends on neuronal excitation. J Neurosci 16:2585-2591.

Menz MD, Ye P, Firouzi K, Nikoozadeh A, Pauly KB, Khuri-Yakub P, Baccus SA (2019) Radiation force as a physical mechanism for ultrasonic neurostimulation of the ex vivo retina. J Neurosci 39:6251-6264.

Min BK, Bystritsky A, Jung KI, Fischer K, Zhang Y, Maeng LS, In Park S, Chung YA, Jolesz FA, Yoo SS (2011) Focused ultrasound-mediated suppression of chemically-induced acute epileptic EEG activity. BMC Neurosci 12:23.

Miocinovic S, Somayajula S, Chitnis S, Vitek JL (2013) History, applications, and mechanisms of deep brain stimulation. JAMA Neurol 70:163-171.

Money TGA, Rodgers CI, McGregor SMK, Robertson RM (2009) Loss of potassium homeostasis underlies hyperthermic conduction failure in control and preconditioned locusts. J Neurophysiol 102:285-293.

Nicholls J, Baylor D (1968) Specific modalities and receptive fields of sensory neurons in CNS of the leech. J Neurophysiol 31:740-756.
Olsen $\varnothing$, Calabrese R (1996) Activation of intrinsic and synaptic currents in leech heart interneurons by realistic waveforms. J Neurosci 16:4958-4970.

Ort CA, Kristan WB Jr, Stent GS (1974) Neuronal control of swimming in the medicinal leech. II. Identification and connections of motor neurons. J Comp Physiol 94:121-154.

Pietrobon D, Moskowitz MA (2014) Chaos and commotion in the wake of cortical spreading depression and spreading depolarizations. Nat Rev Neurosci 15:379-393.

Plaksin M, Shoham S, Kimmel E (2014) Intramembrane cavitation as a predictive bio-piezoelectric mechanism for ultrasonic brain stimulation. Phys Rev X 4.

Prieto ML, Firouzi K, Khuri-Yakub BT, Maduke M (2018) Activation of Piezo1 but not NaV1.2 channels by ultrasound at $43 \mathrm{MHz}$. Ultrasound Med Biol 44:1217-1232.

Prieto ML, Firouzi K, Khuri-Yakub BT, Madison DV, Maduke M (2020) Spike frequency-dependent inhibition and excitation of neural activity by high-frequency ultrasound. J Gen Physiol 2:152.

Puhl J, Mesce KA (2008) Dopamine activates the motor pattern for crawling in the medicinal leech. J Neurosci 28:4192-4200.

Raghavan P (2018) Emerging therapies for spastic movement disorders. Phys Med Rehabil Clin N Am 29:633-644.

Rinaldi PC, Jones JP, Reines F, Price LRR (1991) Modification by focused ultrasound pulses of electrically evoked responses from an in vitro hippocampal preparation. Brain Res 558:36-42.

Rodgers Cl, Armstrong GAB, Shoemaker KL, LaBrie JD, Moyes CD, Robertson RM (2007) Stress preconditioning of spreading depression in the locust CNS. PLoS One 2:e1366.

Romanenko S, Siegel PH, Wagenaar DA, Pikov V (2014) Effects of millimeter wave irradiation and equivalent thermal heating on the activity of individual neurons in the leech ganglion. J Neurophysiol 112:2423-2431.

Sato T, Shapiro MG, Tsao DY (2018) Ultrasonic neuromodulation causes widespread cortical activation via an indirect auditory mechanism. Neuron 98:1031-1041.

Schneider ER, Anderson EO, Gracheva EO, Bagriantsev SN (2014) Temperature sensitivity of two-pore (K2P) potassium channels. Curr Top Membr 74:113-133.

Shapiro MG, Homma K, Villarreal S, Richter CP, Bezanilla F (2012) Infrared light excites cells by changing their electrical capacitance. Nat Commun 3:736

Shealy CN, Henneman E (1962) Reversible effects of ultrasound on spinal reflexes. Arch Neurol 6:374-386.

Somjen GG (2001) Mechanisms of spreading depression and hypoxic spreading depression-like depolarization. Physiol Rev 81:1065-1096.

Spong KE, Andrew DR, Robertson MR (2016) Mechanisms of spreading depolarization in vertebrate and insect central nervous systems. J Neurophysiol 116:1117-1127.

St. John Smith E (2018) Advances in understanding nociception and neuropathic pain. J Neurol 265:231-238.

Stuart AE (1970) Physiological and morphological properties of motoneurones in the central nervous system of the leech. J Physiol 209:627-646.

Takagi SF, Higashino S, Shibuya T, Osawa N (1960) The actions of ultrasound on the myelinated nerve, the spinal cord and the brain. Jpn J Physiol 10:183-193.

Tsui PH, Wang SH, Huang CC (2005) In vitro effects of ultrasound with different energies on the conduction properties of neural tissue. Ultrasonics 43:560-565.

Tufail Y, Matyushov A, Baldwin N, Tauchmann ML, Georges J, Yoshihiro A, Tillery SIH, Tyler WJ (2010) Transcranial pulsed ultrasound stimulates intact brain circuits. Neuron 66:681-694.

Tyler WJ, Tufail Y, Finsterwald M, Tauchmann ML, Olson EJ, Majestic C (2008) Remote excitation of neuronal circuits using low-intensity, low-frequency ultrasound. PLoS One 3:e3511.

Ueda M, Bures J, Fischer J (1977) Spreading depression elicited by thermal effects of ultrasonic irradiation of cerebral cortex in rats. $J$ Neurobiol 8:381-393. 
Wang H, Wang B, Normoyle KP, Jackson K, Spitler K, Sharrock M, Miller CM, Best C, Llano D, Du R (2014) Brain temperature and its fundamental properties: a review for clinical neuroscientists. Front Neurosci 8:307.

Wright CJ, Rothwell Saffari N (2015) Ultrasonic stimulation of peripheral nervous tissue: an investigation into mechanisms. J Phys Conf Ser 581:012003.

Wright C, Haqshenas S, Rothwell J, Saffari N (2017) Unmyelinated peripheral nerves can be stimulated in vitro using pulsed ultrasound. Ultrasound Med Biol 43:2269-2283.

Wu J, Fisher RS (2000) Hyperthermic spreading depressions in the immature rat hippocampal slice. J Neurophysiol 84:1355-1360.

Yoo SS, Bystritsky A, Lee JH, Zhang Y, Fischer K, Min BK, McDannold NJ, Pascual-Leone A, Jolesz FA (2011) Focused ultrasound modulates region-specific brain activity. Neuroimage 56:1267-1275.

Yoo SH, Croce P, Margolin RW, Lee SD, Lee W (2017) Pulsed focused ultrasound changes nerve conduction of earthworm giant axonal fibers. Neuroreport 28:229-233.

Yoo S, Mittelstein DR, Hurt R, Lacroix J, Shapiro MG (2020) Focused ultrasound excites neurons via mechanosensitive calcium accumulation and ion channel amplification. bioRxiv. doi: https://doi. org/10.1101/2020.05.19.101196.

Zhou W, Wang J, Wang K, Huang B, Niu L, Li F, Cai F, Chen Y, Liu X, Zhang X, Cheng H, Kang L, Meng L, Zheng H (2017) Ultrasound neuromodulation chip: activation of sensory neurons in: Caenorhabditis elegans by surface acoustic waves. Lab Chip 17:1725-1731. 